\title{
Langmuir-Blodgett Membranes of 13-Aryltrideca-10,12-diynoic Acids
}

\author{
María del Pilar Carreón, Guillermina Burillo, Lioudmila Fomina,* \\ and Takeshi Ogawa ${ }^{* \dagger}$ \\ Instituto de Ciencias Nucleares, Universidad Nacional Autónoma de México, Circuito Exterior, \\ Ciudad Universitaria, México DF 04510, México \\ * Instituto de Investigaciones en Materiales, Universidad Nacional Autónoma de México, Circuito Exterior, \\ Ciudad Universitaria, México DF 04510, México
}

(Received May 15, 1997)

\begin{abstract}
To study the formation and properties of Langmuir-Blodgett membranes of aromatic diacetylenes, 4 novel diacetylenics amphiphiles were synthesized, and their multilayer membranes were prepared and photopolymerization was carried out. 13-Phenyl-10,12-tridecynoic acid did not give a stable isotherm, and the structure appeared not proper for topochemical polymerization of the diacetylenic group, but substitution on the para-position of the acids stabilized the isotherm giving multilayer $\mathrm{Y}$ type membranes whose diacetylene groups readily polymerized on irradiation of UV light. There was a clear relationship between electronic density of aromatic groups, absorption maxima of polydiacetylene films and resistance to radiation.
\end{abstract}

KEY WORDS Aromatic Diacetylenes / Langmuir-Blodgett Membranes / Photo-Polymerization /

Polydiacetylenes (PDAs) belong to a class of polymers which consist of a unique conjugation of single-doublesingle-triple linkages, and because of their crystalline nature and third order nonlinear optical properties, they attract much attention. However, PDAs are polymers whose processing to desired forms is not at all simple, and various methods have been attempted. One conventional method is the Langmuir-Blodgett (LB) membrane technique. A variety of diacetylenic amphiphiles has been synthesized and studied systematically. Tieke and Wegner's group extensively studied the diacetylene polymerization in LB films. ${ }^{1-4}$ They discuss mainly the relationships between the chemical structure, polymerizability of the diacetylenic groups, stability of the LB films, orientation of the molecules and packing of the hydrophobic chains.

However, all amphiphilic diacetylenes so far studied are aliphatic diacetylenes. In these cases the conjugation range is limited only to PDA back bones. If aromatic diacetylenes (DAs) are used, the resulting PDAs have more extended conjugation and nonlinear optical (NLO) properties may be improved. The present authors for the first time reported the synthesis and formation of LB membranes of aromatic diacetylenes. ${ }^{5}$ Thus, blue films of PDAs were obtained by irradiation of UV light from $m$-alca-1,3-diynylbenzoic acids.

In this work, a series of novel amphiphilic aromatic diacetylenes were synthesized, and the formations of LB membranes were investigated as well as polymerizability.

\section{EXPERIMENTAL}

\section{Materials}

The amphiphilic DA monomers were synthesized according to the reactions shown in Scheme 1. The synthetic procedures of the compounds are described below:

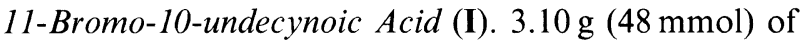

\footnotetext{
$\uparrow$ To whom correspondence should be addressed.
}

bromine were added to $30 \mathrm{ml}$ of aqueous sodium hydroxide solution $(0.110 \mathrm{~mol} \mathrm{NaOH})$ at $5-10^{\circ} \mathrm{C}$ and then $5 \mathrm{~g}(27.5 \mathrm{mmol})$ of 10 -undecynoic acid dissolved in $50 \mathrm{ml}$ of aqueous sodium hydroxide solution $(27.5 \mathrm{mmol})$ were added at room temperature, and the mixture was stirred for $24 \mathrm{~h} .7 \mathrm{~g}$ of $\mathrm{Na}_{2} \mathrm{SO}_{3}$ dissolved in $100 \mathrm{ml}$ of water, were added to the solution to eliminate $\mathrm{BrO}^{-}$. The solution was acidified with dilute hydrochloric acid, and the precipitate was filtered, washed with water and dried in vacuum; yield $90 \%, \mathrm{mp} 40^{\circ} \mathrm{C}$.

13-Aryltrideca-10,12-diynoic Acids. Under nitrogen atmosphere were mixed $10.34 \mathrm{mmol}$ of phenyl acetylene, excess $(200 \%) 50 \%$ aqueous solution of butylamine, $10 \mathrm{ml}$ ethanol, catalytic amount of copper(I) chloride, and small amount of hydroxylamine hydrochloride. The mixture was stirred at room temperature for $5 \mathrm{~min}$, and $11.49 \mathrm{mmol}$ of I dissolved in excess ethanol were added dropwide with stirring. The reaction was exothermic and the reaction flask was cooled occasionally with an ice bath to avoid excess temperature rise. The mixture was stirred at room temperature for $24 \mathrm{~h}$. The mixture was then poured into $200 \mathrm{ml}$ water, and acidified with dilute hydrochloric acid. The insoluble product was filtered,

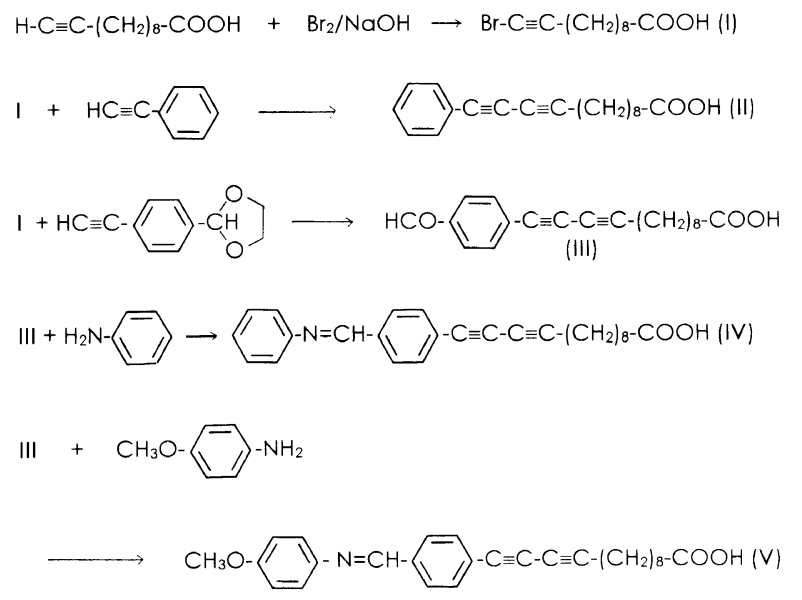

Scheme 1. Synthetic route to 13-aryltrideca-10,12-diynoic acids. 
Table I. 13-Aryltrideca-10,12-diynoic acids

\begin{tabular}{|c|c|c|c|c|}
\hline \multicolumn{2}{|c|}{ Compound } & \multirow{3}{*}{$\begin{array}{l}\mathrm{mp} \\
{ }^{\circ} \mathrm{C}\end{array}$} & \multicolumn{2}{|c|}{ Elemental analysis } \\
\hline & $\mathrm{C}-\left(\mathrm{CH}_{2}\right)_{8}-\mathrm{COOH}$ & & \multirow{2}{*}{$\frac{\text { Found }}{\%}$} & \multirow{2}{*}{$\frac{\text { Calcd }}{\%}$} \\
\hline $\mathrm{R}$ & Code & & & \\
\hline \multirow[t]{2}{*}{$-\mathrm{H}$} & $\phi \mathrm{DA}$ & 81 & $\mathrm{C}: 80.58$ & C: 80.56 \\
\hline & & & $\mathrm{H}: 8.12$ & $\mathrm{H}: \quad 8.13$ \\
\hline \multirow[t]{2}{*}{$-\mathrm{CHO}$} & $\phi \mathrm{DACHO}$ & 76 & C: 76.91 & C: 76.92 \\
\hline & & & $\mathrm{H}: \quad 7.60$ & H: 7.69 \\
\hline \multirow{3}{*}{$-\mathrm{CH}=\mathrm{N}-\mathrm{C}$} & $\phi \mathrm{DAC}=\mathrm{N}$ & 70 & $C: 81.23$ & $C: 81.25$ \\
\hline & & & $\mathrm{H}: \quad 6.76$ & $\mathrm{H}: \quad 6.77$ \\
\hline & & & $\mathrm{N}: \quad 4.50$ & $\mathrm{~N}: \quad 4.49$ \\
\hline \multirow{3}{*}{$-\mathrm{CH}=\mathrm{N}-2$} & $\phi \mathrm{DAC}=\mathrm{NOCH}_{3}$ & 65 & $\mathrm{C}: 78.08$ & C: 78.07 \\
\hline & & & $\mathrm{H}: \quad 7.00$ & $\mathrm{H}: \quad 6.99$ \\
\hline & & & $\mathrm{N}: \quad 3.37$ & $\mathrm{~N}: \quad 3.37$ \\
\hline
\end{tabular}

washed with water, and dried in vacuum, yield $91 \%$. Under nitrogen atmosphere were mixed $10.34 \mathrm{mmol}$ of 2-[4'-(ethynyl)phenyl]-1,3-dioxolane, an excess (200\%) $50 \%$ aqueous solution of butylamine, $10 \mathrm{ml}$ ethanol, catalytic amount of copper(I) chloride, and small amount of hydroxylamine hydrochloride. The mixture was stirred at room temperature for $5 \mathrm{~min}$, and $11.49 \mathrm{mmol}$ of I dissolved in excess ethanol were added dropwise with stirring. The reaction was exothermic and the reaction flask was cooled occasionally with an ice bath. The mixture was stirred at room temerature for $24 \mathrm{~h}$ and poured into $200 \mathrm{ml}$ water, and acidified with dilute hydrochloric acid. The insoluble product was filtered, washed with water, and dried in vacuum, yield $70 \%$.

$5 \mathrm{mmol}$ of (III) were dissolved in $20 \mathrm{ml}$ ethanol, and $5 \mathrm{mmol}$ of aniline were added dropwise with stirring. The mixture was stirred at room temperature for $4 \mathrm{~h}$. The insoluble product was filtered, washed with ethanol, and dried in vacuum, yield $75 \%$.

$5 \mathrm{mmol}$ of (III) were dissolved in $20 \mathrm{ml}$ ethanol, and 5 mmol of $p$-anisidine were added dropwise with stirring. The mixture was stirred at room temperature for $4 \mathrm{~h}$. The insoluble product was filtered, washed with ethanol and dried in vacuum; yield $75 \%$.

Results of elemental analysis are shown in Table I.

\section{Formation of LB Films}

Monolayers were spread from a $\mathrm{CHCl}_{3}$ ( $\mathrm{HPLC}$ grade, Aldrich) solution $\left(1 \mathrm{mg} \mathrm{ml}^{-1}\right)$ on the surface of the subphase. Subphase water was triply distilled, and $\mathrm{CdCl}_{2}$ $\left(1 \times 10^{-4} \mathrm{M}\right)$ was added to stabilize the monolayer and $\mathrm{pH}$ of the subphase became 6.0. Pressure-area isotherms were measured at a compression speed of $5 \mathrm{~mm} \mathrm{~min}^{-1}$, using a Miyata-type moving wall LB film deposition apparatus, Model NL-LB240-MWA, manufactured by Nippon Laser \& Electronic Laboratory.

The monomolecular films were successively transferred by LB technique onto ultra-pure quartz plates previously treated $^{6}$ with mixture of sulfuric acid-nitric acid $(1: 1)$ for $30 \mathrm{~min}$ at a temperature of about $370 \mathrm{~K}$ and neutralized with $2.5 \times 10^{-4} \mathrm{M}$ solution of sodium hydroxide, rinsed thoroughly with triply distilled water and dried in a desiccator. Deposition was carried out at room temperature by up-and-down trips to form Y-layers under a constant surface pressure of $10 \mathrm{mN} \mathrm{m}^{-1}$ for $\phi \mathrm{DA}$, and $35 \mathrm{mN} \mathrm{m}^{-1}$ for $\phi \mathrm{DACHO}, \phi \mathrm{DAC}=\mathrm{N}$, and $\phi \mathrm{DAC}=\mathrm{NOCH}_{3}$ at a deposition rate of $5 \mathrm{~mm} \mathrm{~min}^{-1}$ for the four cases.

\section{Polymerization}

Polymerization of diacetylenic groups was carried out by UV irradiation using a $450 \mathrm{~W}$ medium-pressure mercury lamp supplied by Ace Glass Inc., at a distance of $30 \mathrm{~cm}$ from the light source under air atmosphere.

\section{Multilayer Characterization}

Membrane thickness was calculated from ellipsometry data. Ellipsometry was performed with an Auto EIIII automated ellipsometer manufactured by Rudolph Research. With this instrument, elliptically polarized $6328 \AA$ wavelength light strikes the sample at an angle of $70^{\circ}$ from vertical. The accuracy of the readings was enhanced using the two-zone averaging.

FT-IR spectra of the films deposited onto a germanium plate were taken using a Perkin-Elmer 1600 using Attenued Total Reflection (ATR) mode. The spectra were collected by scanning each sample 256 times with a resolution of $4 \mathrm{~cm}^{-1}$.

UV-VIS absorption spectra were recorded using a Perkin-Elmer 553 Fast Scan spectrometer.

\section{RESULTS AND DISCUSSION}

\section{Monolayers}

Figure 1 shows surface pressure-area isotherms of the compounds which require cadmium ions in the subphase to form a stable condensed monolayer. The addition of cadmium ion to the aqueous subphase had different results as shown in Figure 1. In the absence of cadmium ions, stable monolayers could not be obtained. A gradual increase of the surface pressure was observed for $\phi \mathrm{DA}$, over the area from 25 to $40 \AA^{2}$ molecule $^{-1}$, indicating the monolayer to be stable as compared with the case where the subphase was only pure water. Stable monolayers with good condensed phase were obtained for $\phi \mathrm{DACHO}, \phi \mathrm{DAC}=\mathrm{N}$, and $\phi \mathrm{DAC}=\mathrm{NOCH}_{3}$, and extrapolated areas to zero pressure had $20-23 \AA^{2}$ molecule $^{-1}$, whereas $\phi \mathrm{DA}$ had an area of $32 \AA^{2}$ molecule $^{-1}$. In all four cases there were observed collapse pressures, being $15 \mathrm{mN} \mathrm{m}^{-1}$ for $\phi \mathrm{DA}$, and $55-60 \mathrm{mN} \mathrm{m}^{-1}$ for $\phi \mathrm{DACHO}, \phi \mathrm{DAC}=\mathrm{N}$, and $\phi \mathrm{DAC}=\mathrm{NOCH}_{3}$. In all the cases, deposition could be satisfactorily carried out at a transfer ratio of $0.95-1.00$, which indicates the formation of Y-type membranes.

The ellipsometry results are shown in Table II. Although there is a certain inherent error in the ellipsometry, the observed thickness was considered acceptable.

\section{Photopolymerization of Membranes}

Figure 2 shows the absorption spectra of a 40-layers membrane of $\phi \mathrm{DA}$ with different irradiation times. The absorbance of the broad peak at around $540 \mathrm{~nm}$ increased with advance of polymerization. The broad shape of the absorption spectra indicates that the crystalline structure of the DA molecules in the layer is not ideal for topochemical polymerization, and this is quite different from aromatic diacetylenic membranes where the aromatic ring is on the water surface. ${ }^{5}$ However, since the unirradiated membrane had no absorption over the 

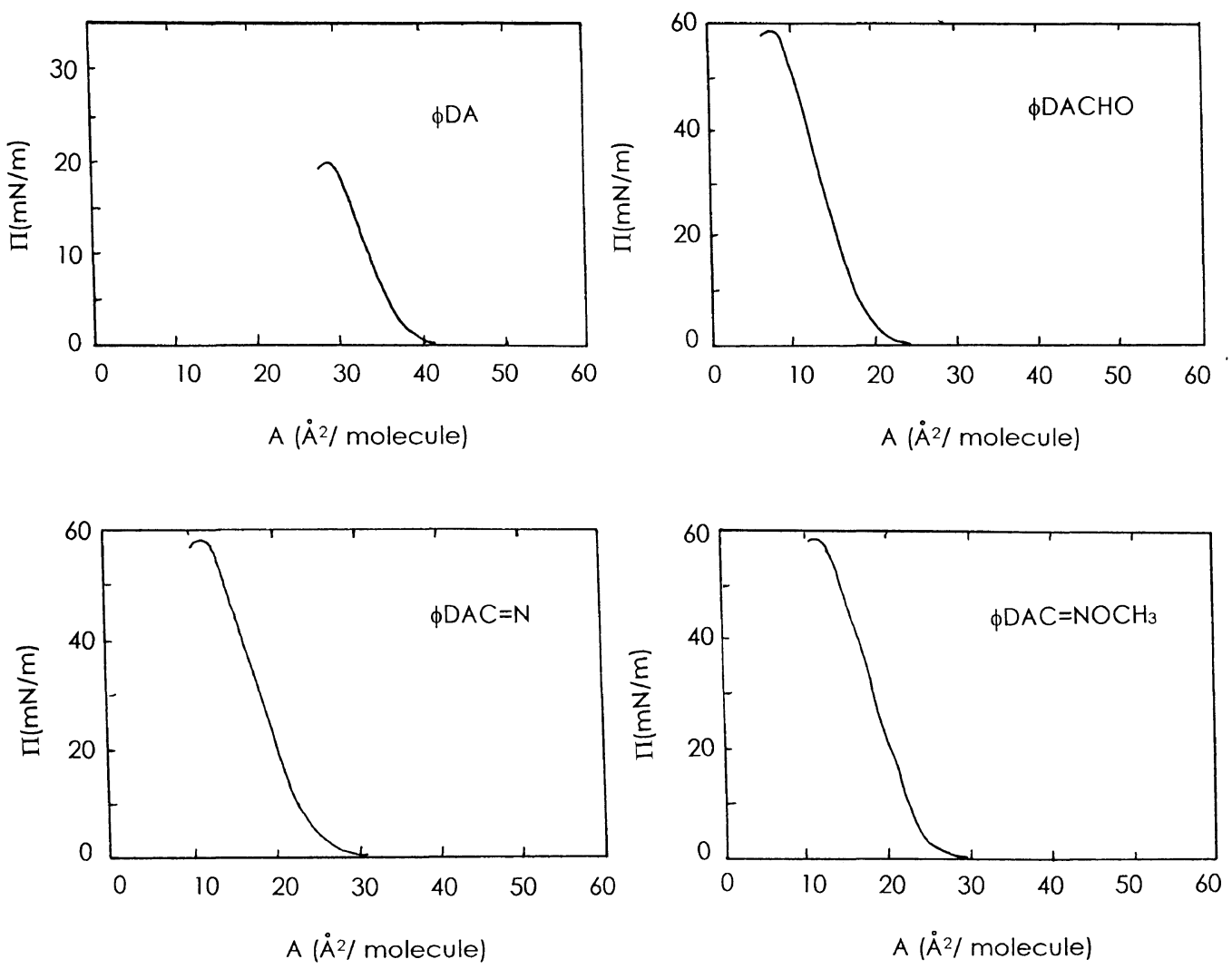

Figure 1. Surface pressure-area isotherms of 13 -aryltrideca-10,12-diynoic acids for aqueous solution of $\mathrm{CdCl}_{2}\left(1 \times 10^{-4} \mathrm{M} ; \mathrm{pH}^{6.0)}\right.$.

Table II. Monolayer thickness

\begin{tabular}{lc}
\hline \multicolumn{1}{c}{ Code } & Thickness per layer $/ \AA$ \\
\hline DA & $25.1 \pm 3$ \\
$\phi$ DACHO & $26.9 \pm 2$ \\
$\phi$ DAC $=\mathrm{N}$ & $28.7 \pm 5$ \\
$\phi \mathrm{DAC}=\mathrm{NOCH}_{3}$ & $30.9 \pm 4$ \\
\hline
\end{tabular}

visible region, the polymerization apparently took place.

Figure 3 illustrates the dependence of absorption spectra of a 40-layers membrane of $\phi \mathrm{DACHO}$ on UVlamp irradiation time. The unirradiated membrane had no absorption in the visible region. Contrary to the $\phi D A$ case, two absorption peaks typical of topochemically polymerized PDAs, appeared at 568 and $610 \mathrm{~nm}$. It seems that the carbonyl group significantly contributes to the formation of crystal structures favorable for topochemical polymerization. The absorption maxima of poly $(\phi \mathrm{DACHO})$ were somewhat lower compared to those of other PDA LB membranes reported previous$1 y^{2,4,5}$ (at around 600 and $640 \mathrm{~nm}$ ), and this is probably due to the electron withdrawing aldehyde group. Polymerization appeared to be completed with $60 \mathrm{~min}$ of irradiation (Figure 4), and absorption intensity began to decrease, indicating that the polymer film is possibly photodegradated by oxidation. This phenomenon of oxidation during irradiation was observed for diacetylenic benzoic acids. ${ }^{1,5}$

Figure 4 shows the FT-IR spectra of $\phi$ DACHO before and after UV irradiation, at the region where acetylenic and diacetylenic stretching appear. Two bands corresponding to the diacetylenic groups before the UV irradiation disappeared completely, and a new acetylenic

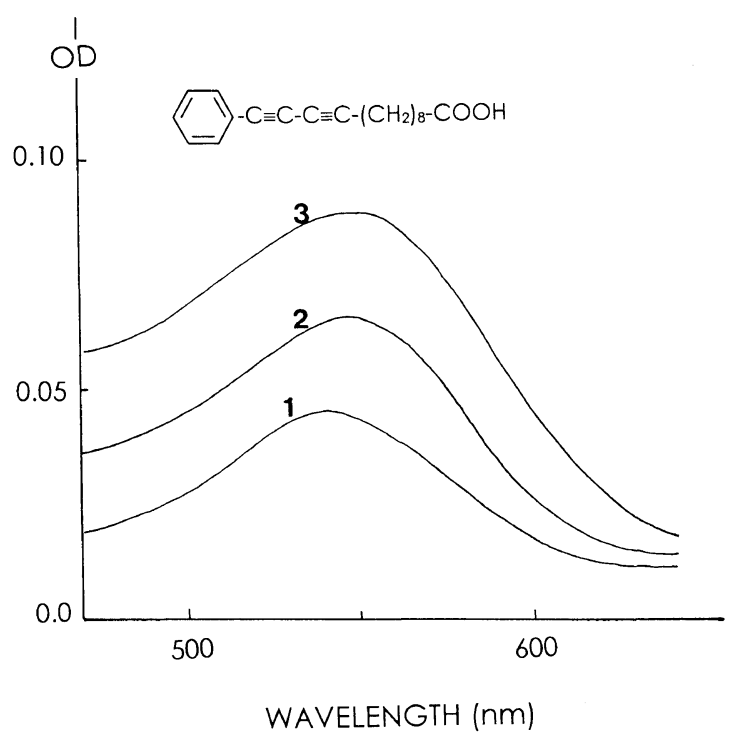

Figure 2. Visible absorption spectra of 40-layer LB membranes of $\phi$ DA irradiated with UV light for different periods. 1, $5 \mathrm{~min} ; 2,10 \mathrm{~min}$; $3,30 \mathrm{~min}$.

band appeared at $60 \mathrm{~min}$ irradiation. Therefore, topochemical polymerizaton took place.

Figure 5 shows changes in the absorption spectrum for the 40-layers membrane of $\phi D A C=N$ irradiated for different periods. No absorption was observed in visible region for the unirradiated membrane. Two absorption peaks appeared as usual at 600 and $640 \mathrm{~nm}$, as the carbonyl group was changed to the less electronwithdrawing azomethyn group. The absorbance intensity of the film increased with irradiation time until $60 \mathrm{~min}$, 


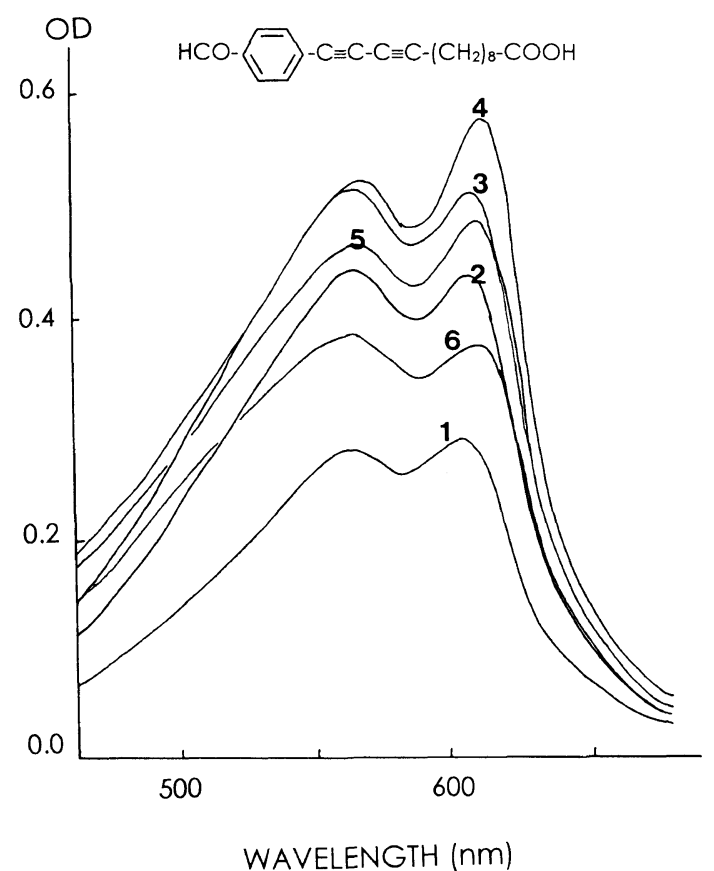

Figure 3. Visible absorption spectra of 40-layer LB membranes of $\phi \mathrm{DACHO}$ irradiated with UV light for different periods. 1, $10 \mathrm{~min}$; 2, $20 \mathrm{~min} ; 3,30 \mathrm{~min} ; 4,60 \mathrm{~min} ; 5,100 \mathrm{~min} ; 6,120 \mathrm{~min}$.

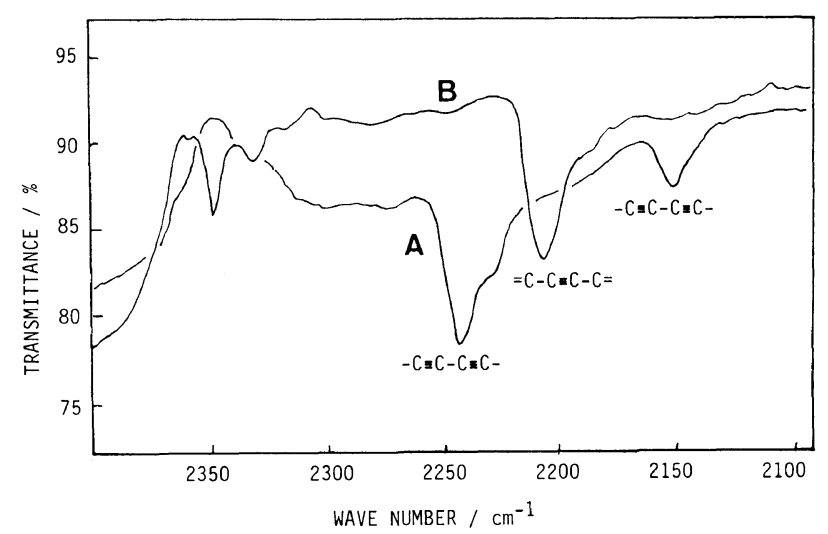

Figure 4. FT-IR spectra of 40-layer membranes of $\phi$ DACHO before and after UV irradiation for $60 \mathrm{~min}$. A, before; B, after irradiation.

but after $60 \mathrm{~min}$, absorbance peaks started to blue shift and became broader, indicating photodegradation of the conjugated chains of polydiacetylene by oxidation.

Figure 6 illustrates changes of absorption spectra of 40-layers membrane of $\phi \mathrm{DAC}=\mathrm{NOCH}_{3}$ with irradiation time. Two absorption peaks characteristic of polydiacetylenes, appeared at 645 and $685 \mathrm{~nm}$. This red shift is due to the methoxy group which increases electronic density in the PDA chains. The absorbance of the multilayer increased with irradiation time up to $40 \mathrm{~min}$, and with irradiation for more than $40 \mathrm{~min}$, absorption maxima decreased as seen in the previous cases, indicating that the polymer is photodegraded due to oxidation. This PDA is more vulnerable to oxidation than others which have less electronic density.

The blue shift of the absorption peaks with prolonged irradiation is thought due to oxidation. The photocrosslinking of polydiacetylene chains cannot be eliminated. However, a powerful UV lamp forms ozone of

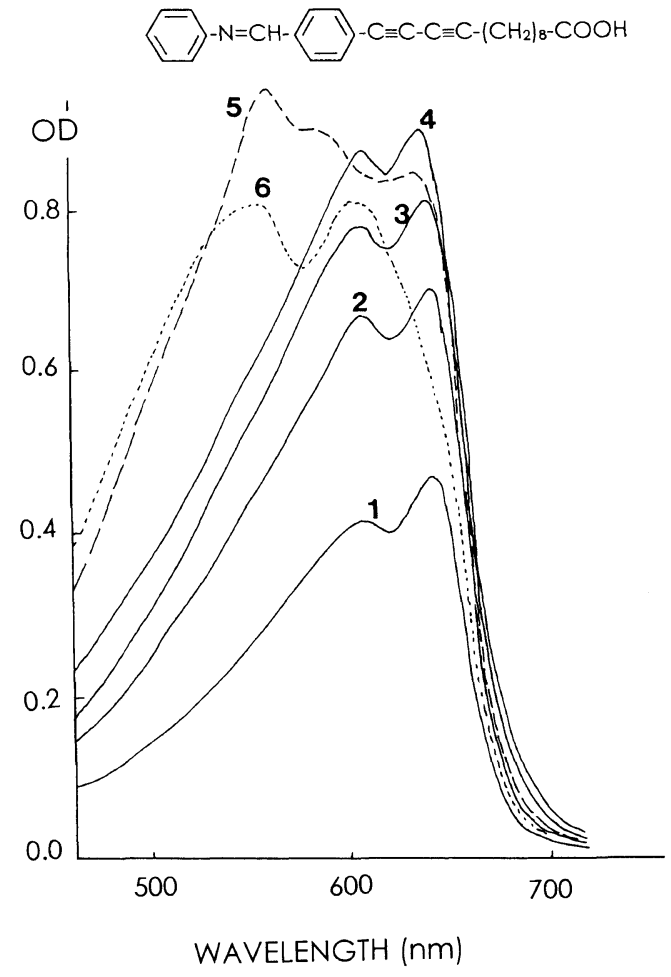

Figure 5. Visible absorption spectra of 40-layer LB membranes of $\phi \mathrm{DAC}=\mathrm{N}$ irradiated with $\mathrm{UV}$ light for different periods. $1,10 \mathrm{~min}$; 2, $20 \mathrm{~min}$; 3, $30 \mathrm{~min}$; 4, $60 \mathrm{~min}$;, $100 \mathrm{~min}$; 6, $150 \mathrm{~min}$.

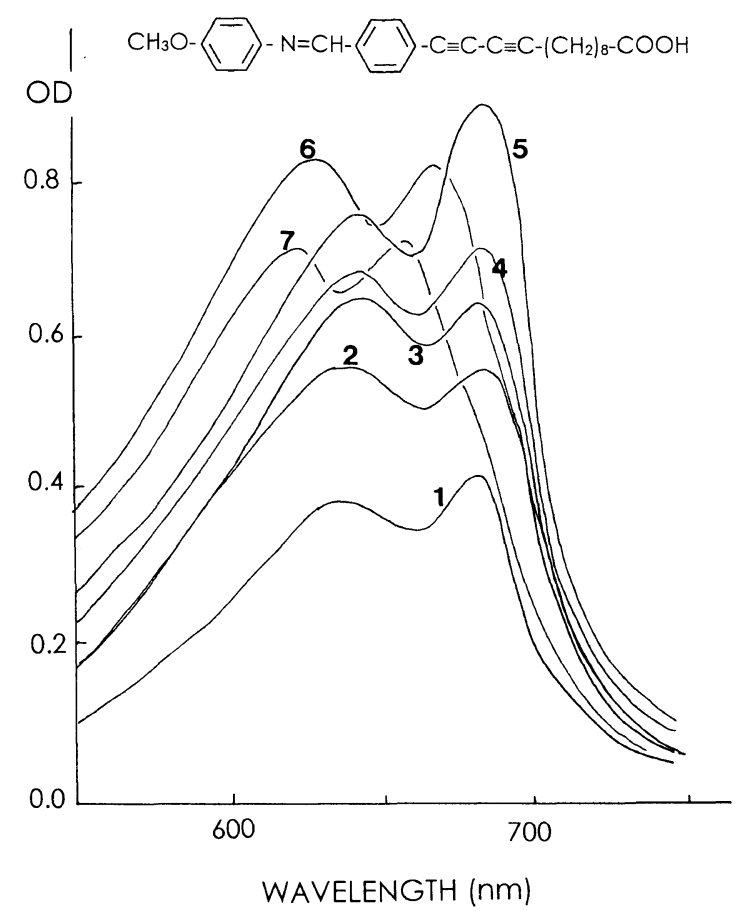

Figure 6. Visible absorption spectra of 40-layer LB membranes of $\phi \mathrm{DAC}=\mathrm{NOCH}_{3}$ irradiated with UV light for different periods. 1, $5 \mathrm{~min} ; 2,10 \mathrm{~min} ; 3,20 \mathrm{~min} ; 4,30 \mathrm{~min} ; 5,40 \mathrm{~min} ; 6,60 \mathrm{~min} ; 7,100 \mathrm{~min}$.

irradiated area, and photo-excited polydiacetylene molecules may contribute to formation of singlet oxygen. Therefore, oxidation is thought a more probable reason for degradation. The resistance toward photodegradation seemed to depend on the electron density of polydiacetylene chains; the higher the electron density, the lower the resistance. 
Third order non-linear optical susceptibility, $\chi^{(3)}$, values reported in literature for LB membranes of polydiacetylenes are quite low, around $10^{-12}$ esu. $^{7}$ The multilayer films of diacetylenic benzoic acids ${ }^{8}$ also gave a low $\chi^{(3)}$ of the same order as reported. ${ }^{7}$ The low observed $\chi^{(3)}$ values may be due to damage caused by laser beam during determination of $\chi^{(3)}$.

\section{CONCLUSIONS}

These results indicate that substitution on the terminal benzene ring stabilize the layer probably by the dipoledipole interaction ( $\mathrm{CHO}$ or $\mathrm{CH}=\mathrm{N}$ groups), and this also gives rise to favorable orientation of the diacetylenic groups for topochemical polymerization. The donor group on the para-position of the terminal benzene ring shifts absorption maxima of polydiacetylenes towards the longer wavelength region (red shift): PhenylCHO- $-(568$ and $610 \mathrm{~nm})<$ Phenyl $-\mathrm{N}=\mathrm{CH}-(600$ and $640 \mathrm{~nm})<\mathrm{CH}_{3} \mathrm{O}-$ Phenyl- $\mathrm{N}=\mathrm{CH}-(645$ and $685 \mathrm{~nm})$. At the same time resistance to oxidation decreased with electron donor groups. Irradiation under an inert atmosphere is being carried out and results will be reported in the future together with third order NLO properties of these films.

Acknowledgment. This work was partially supported by the project of CONACYT (Consejo Nacional de Ciencias y Tecnología) with Contract Number 4264E9406. Thanks are also due to DGAPA (Direccion General de Asuntos de Personal Acedémico) of our university for providing a scholarship to Ma. del Pilar Carreón for her doctorate study.

\section{REFERENCES}

1. B. Tieke, G. Lieser, and G. Wegner, J. Polym. Sci., Polym. Chem. Ed., 17, 1631 (1979).

2. G. Tieke and G. Lieser, J. Colloid Interface Sci., 88, 471 (1982).

3. G. Lieser, G. Tieke, and H. Wegner, Thin Solid Films, 68, 77 (1980).

4. B. Tieke and G. Lieser, Macromolecules, 18, 327 (1985).

5. M. P. Carreón, G. Burillo, V. Agabekov, and T. Ogawa, Polym. J., 29, 103 (1997).

6. G. L. Gaines, Jr., J. Colloid Interface Sci., 54, 430 (1976).

7. J. L. Bredás and R. De Silby, in "Conjugated Polymers," F. Kajzar and J. Messier, Ed., "Third Order Nonlinear Optical Effects in Conjugated Polymers," Kluwer Academic Publishers, Dordrecht, 1991, p 541.

8. Unpublished results. 